\title{
De la evaluación informal a la evalua- ción fundamentada: Reconocer el rol del enseñante en clase
}

Kenneth P. Wolf

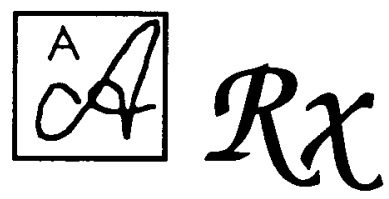

Si las evaluaciones realizadas por los educadores directamente implicados están sujetas a limitaciones $y$ errores, las evaluaciones "formales" $y$ externas presentan también graves, aunque distintas insuficiencias. El autor propone desde abi nuevas estrategias, métodos, condiciones a cumplir para que la evaluación basada en el educador pueda ser un instrumento potente. Wolf examina estrategias que ayudan a los enseñantes a que la evaluación cotidiana sea más significativa y creíble.

\section{IMAGINEMOS DOS SITUACIONES}

a) La Sra. Knight andaba lentamente por su clase, leyendo el folleto doblado que llevaba en la mano. Su voz era clara y mesurada. Sus alumnos aguardaban la pregunta al final de cada pasaje y luego bajaban la cabeza para mirar sus ejercicios y rellenar con pequeños círculos sus hojas de respuesta con sus lápices del $\mathrm{N}^{\circ} 2$ recién afilados. La Sra. Knight observó que Tod, uno de sus alumnos, estaba haciendo dibujitos en su papel de borrador. Suspiró y le recordó que se concentrara. Al final del ejercicio, recogió los papeles de los alumnos y los remitió a la oficina. Aunque estaría meses esperando los resultados oficiales, abrigaba pocas esperanzas en relación a Tod.

b) La Sra. Knight sabía que la escritura de Tod carecía de organización y que su lectura oral era vacilante. Sus puntuaciones en las pruebas normalizadas eran muy bajas. Sin embargo, al estudiar los trabajos de dibujo de la carpeta de Tod, vio que era capaz de transmitir una pro- 
funda comprensión de ideas y temas complejos a través de sus dibujos. En un dibujo particular, Tod capturó el tema de los "ciclos" ilustrando el "ciclo vital" de una vasija de cerámica Anasazi desde las manos del antiguo artesano que le dio forma, pasando por las manos del niño que la dejó caer, hasta las manos del arqueólogo que reconstruyó la vasija siglos después. La Sra. Knight decidió elaborar más enseñanzas en torno al arte de Tod, animándole a utilizar su talento artístico como puente para desarrollar sus aptitudes para el lenguaje oral y escrito.

Estas dos situaciones de clase acabadas de citar presentan dos puntos de vista opuestos de la evaluación. El primero describe una evaluación formal, término comúnmente utilizado para describir evaluaciones como pruebas normalizadas que están diseñadas y son puntuadas por personas ajenas a la clase. El segundo describe una evaluación informal, etiqueta que suele hacer referencia a evaluaciones elaboradas y aplicadas por los enseñantes en clase, e incluye actividades tales como conversaciones, observaciones y revisiones de los productos de los alumnos.

El empleo del término "informal" para describir las evaluaciones que llevan a cabo los enseñantes en sus clases sugiere que estas evaluaciones son subjetivas, caprichosas, casuales y, quizá, hasta engañosas. En cambio, etiquetar de "formales" las evaluaciones conducidas por personas externas a la clase implica que se trata de evaluaciones objetivas, fiables, científicas y, sin duda, precisas. El resultado final es que las evaluaciones que los enseñantes hacen de sus alumnos son descartadas por las personas ajenas a la clase ( $y$ hasta por muchos enseñantes) por considerarlas no fiables, mientras que las evaluaciones impuestas externamente son consideradas pruebas "verdaderas" de lo que los alumnos saben y pueden hacer.

Sin embargo, se da un reconocimiento cada vez mayor de las limitaciones de utilizar evaluaciones formales para medir el logro de los estudiantes (Archbald y Newmann, 1988; Report of the National Commision on Testing and Public Policy, 1990). Además, cada vez hay más evidencias de que estas pruebas minan la motivación del alumno (Paris, Lawton, Turner y Roth, 1991), desprofesionalizan a los enseñantes (Smith, 1991), trivializan los objetivos curriculares (McNeil, 1988), distorsionan las prácticas educativas (Haertel, 1990) y representan mal el logro de los alumnos (Cannell, 1987).

Al mismo tiempo, el respeto para la evaluación informal está en alza, tanto para mejorar la enseñanza y el aprendizaje en el aula (Calfee y Hiebert, 1989; Goodman, 1991) como para informar a gran escala (Brewer, 1990). Muchos han llegado a creer que la información más válida y valiosa sobre el aprendizaje de los estudiantes no procede de "instantáneas" aisladas y fuera de contexto de su rendimiento, sino de las personas que, dia a día, trabajan estrechamanente con los estudiantes.

Teniendo en consideración todos estos puntos, quisiera proponer una etiqueta alternativa para la evaluación de estudiantes basada en el enseñante, una etiqueta que transmita respeto por los tipos de evaluaciones cotidianas que los enseñantes conscientes llevan a cabo en sus clases 
y que reconozca que las evaluaciones hechas por los enseñantes ofrecen la información más precisa y útil sobre el logro de los alumnos. Cuando los enseñantes tienen objetivos significativos para la enseñanza y fines claros para la evaluación, y cuando utilizan una variedad de estrategias para observar y documentar el rendimiento de sus alumnos en diversos contextos y a lo largo del tiempo, estos enseñantes no están practicando una evaluación informal sino una evaluación fundamentada.

En los apartados que siguen, definiré la evaluación fundamentada y describiré sus características; luego examinaré las condiciones que se deben dar para que la evaluación basada en el enseñante alcance su pleno potencial.

\section{INFORMACION FUNDAMENTADA EN EL AULA}

La evaluación fundamentada se refiere al proceso en el que participan los enseñantes conscientes cuando observan sistemáticamente y documentan selectivamente el rendimiento de sus alumnos mediante múltiples métodos, en diversos contextos y a lo largo del tiempo, a medida que los alumnos participan en actividades de aprendizaje significativas (véase la figura).

El enseñante consciente es la base de la evaluación fundamentada. Cuanto más nos alejemos de evaluaciones publicadas comercialmente, "a prueba de enseñantes", para acercarnos a la evaluación de los alum. nos basada en el enseñante, mayor será la necesidad de que el enseñante tenga conciencia del currículo y la instrucción, de los niños y de su desarrollo, y de los roles que el lenguaje, la cultura y el contexto social desempeñan en el aprendizaje. Para que la evaluación sea fundamentada, los enseñantes deben aplicar este conocimiento para establecer objetivos claros y sólidos para la enseñanza, junto con normas y juegos de pruebas sobre rendimiento cuidadosamente articulados para medir el progreso de los alumnos en relación a estos objetivos.

La observación sistemática de los estudiantes cuando trabajan cuando eligen libros, planifican proyectos, escriben relatos, resuelven problemas y discuten ideas- ofrece a los enseñantes una gran cantidad de información sobre los puntos fuertes, los intereses y las necesidades de los alumnos. Se comenta que Yogi Berra dijo: "Se pueden observar muchas cosas simplemente mirando". Sin embargo, el "mirar a los niños" (Goodman, 1985) debe estar guiado por el conocimiento ya que es difícil ver algo si no sabemos qué estamos buscando.

La observación es esencial pero, sin alguna forma de documentación, gran parte de lo que se ha visto será olvidado o, más probable aún, será recordado de una forma que poco se parecerá al suceso original. Décadas de investigación han ilustrado las debilidades de la memoria humana (Bartlett, 1932; Miller, 1956), pero una documentación en forma de trabajo de los alumnos y de registros del enseñante puede ayudar a compensar estas limitaciones.

En muchas aulas, la documentación adopta la forma de carpetas de alumno (Wolf, 1989). Estas recopilaciones de trabajos y reflexiones de 
alumnos ofrecen un registro visible de los logros de los alumnos y permite, tanto a estudiantes como a enseñantes, ampliar y evaluar el aprendizaje de los alumnos. Además, algunos enseñantes mantienen carpetas de clase (Valencia y Calfee, en prensa) en las que registran sus observaciones de estudiantes individuales y de la clase como conjunto mediante notas escritas, listas de comprobación y perfiles de clase.

Sin embargo, se impone una nota de precaución. Aunque es necesaria una cierta documentación de productos y procesos, recopilar productos de los alumnos y registrar observaciones puede ser una tarea que emplee demasiado tiempo para los enseñantes, y dar demasiado énfasis a la documentación puede interferir en la enseñanza y el aprendizaje. Sin embargo, mediante una consideración cuidadosa de los objetivos de la enseñanza y de los fines de la evaluación, enseñantes y estudiantes pueden ser selectivos en la información que recopilen para que ésta pueda ser significativa y manejable.

Los métodos múltiples de evaluación ofrecen una variedad de ventanas al aprendizaje de los alumnos. Un documento escrito durante una sesión de 45 minutos en respuesta a unas instrucciones estanzarizadas puede ofrecer un punto de vista de la aptitud para la escritura de un estudiante muy distinto al que ofrece una redacción sobre un tema seleccionado por el propio estudiante y que ha pasado por varios borradores. Ningún método único de evaluación es suficiente para comprender todo lo que es importante saber sobre cada estudiante, pero una mezcla de estrategias de evaluación puede ayudar a los enseñantes a elaborar una imagen más completa. Las entrevistas con los estudiantes, las recopilaciones de sus trabajos, las evaluaciones de rendimiento y las pruebas con lápiz y papel son todas fuentes de información potencialmente valiosas.

La evaluación debería darse en contextos diversos porque el rendimiento de un estudiante puede variar mucho en función de los textos, las tareas y las situaciones (Lipson y Wixson, 1986). El mismo estudiante que puede comprender y discutir con profundidad Beloved, de Toni Morrison (Knopf, 1987), puede tener dificultad para comprender y comentar un libro de texto elemental sobre electrónica aunque pueda decodificar todas las palabras (por ejemplo, circuito, voltaje, amperio). De manera similar, el estudiante que puede escribir una cáustica canción rap sobre la injusticia racial puede parecer ser un escritor sofisticado, pero cuando se le da la tarea de escribir una exposición sobre el mismo tema puede ser considerado mucho menos competente.

Reconocer que un estudiante ofrece un gran rendimiento en un contexto pero rinde muy poco en otro, aun cuando el contexto que se evalúe sea similar, sugiere que para que los enseñantes sean capaces de determinar las condiciones para apoyar mejor el desarrollo de sus alumnos necesitan evaluar a sus alumnos en varias situaciones y contextos diferentes. Esto es especialmente cierto en los estudiantes cuyos antecedentes y culturas pueden variar en relación a la cultura mayoritaria en la escuela (Heath, 1983).

La evaluación a lo largo del tiempo es importante porque el aprendizaje es un proceso continuo y dinámico (Valencia, McGinley y Pearson, 
Fxira 1

Modelo de información fundamentada

Emseñante consciente

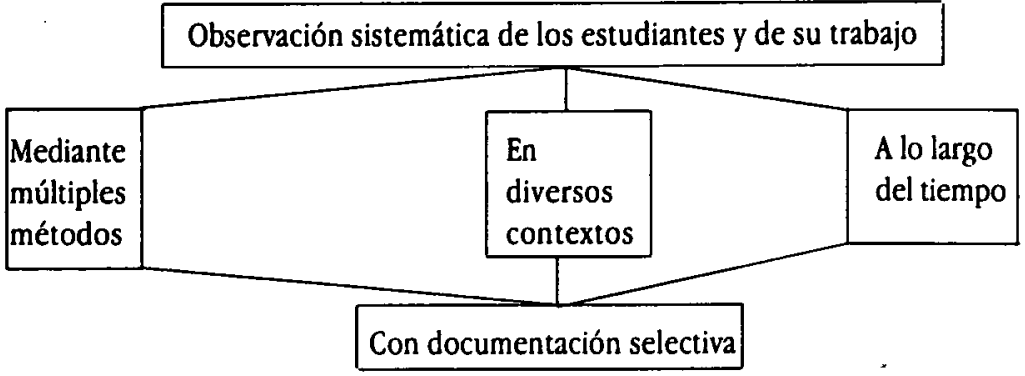

Actividades auténticas

1990). La evaluación continua ofrece a enseñantes y alumnos un feedback regular sobre su rendimiento y les permite ajustar sus actuaciones en consecuencia. Además, algunas características de las actuaciones de los estudiantes no se manifiestan a menos que se contemplen en múltiples ocasiones. El alumno que lee con entusiasmo con su compañero durante los períodos de lectura compartida quizá no merezca una atención especial a menos que el enseñante se dé cuenta de que el companero del alumno lleva a cabo toda la lectura día tras día, o el estudiante que se aplica con esmero a escribir un relato cada día durante el taller de redacción puede que no parezca necesitar ayuda hasta que el enseñante determine que el alumno está revisando continuamente el mismo borrador, una y otra vez. En ocasiones, una pauta solo se hace visible al examinar el rendimiento de un estudiante o de una clase en varias ocasiones distintas.

Observar a los estudiantes mientras participan activamente en actividades de aprendizaje significativas y examinar el trabajo que producen no solo presenta la visión más auténtica y completa de lo que los estudiantes saben y pueden hacer, sino también la más informativa. Sin embargo, en muchas clases tradicionales los tipos de actividades de lectoescritura en que participan los estudiantes son muy limitados.

Si los estudiantes son confinados a una gama estrecha de actividades, las evaluaciones que se realicen darán una imagen de los estudiantes igualmente limitada y los enseñantes tendrán poco acceso a la gama de talentos e intereses de que gozan sus estudiantes. Si los estudiantes trabajan en aislamiento y se dedican a rellenar ejercicios todo el día, el enseñante dispondrá de poca información, por ejemplo, del éxito del alumno en colaborar con otros o de sus preferencias literarias. Como Shulman (1991, comunicación personal) ha observado, es difícil hacer una buena evaluación a partir de un mal currículo.

Sin embargo, si el currículo recalca tanto el proceso como el producto (Atwell, 1987), fomenta una comprensión y una composición de alto nivel (Graves, 1983; Pearson, 1984), valora los objetivos motivacionales 
además de los cognoscitivos (Winograd y Paris, 1989) y permite a los estudiantes seguir vías individuales hacia la lectoescritura (Taylor, 1989), entonces la evaluación que los enseñantes llevan a cabo en sus clases puede ser una rica y valiosa fuente de información para guiar el aprendizaje de los estudiantes e informar sobre él.

\section{APOYO A LA EVALUACION BASADA EN EL ENSEÑANTE FUERA DE CLASE}

Aunque la evaluación basada en el enseñante promete mejorar el aprendizaje de los estudiantes y ofrecer indicadores más auténticos del logro de los alumnos, su impacto será limitado a menos que se tengan en cuenta dos condiciones. En primer lugar, ampliar el número de enseñantes que practican una evaluación fundamentada exigirá una mayor preparación en evaluación dentro de la clase, antes y durante el desempeño profesional. Los enseñantes reciben poca guía, si es que reciben alguna, en el desarrollo y la interpretación de evaluaciones basadas en clase (Stiggins, 1985, 1991). Curiosamente, sin embargo, aunque la evaluación fundamentada basada en el enseñante requiere la presencia de profesionales conscientes en el aula, implicar a los enseñantes en la evaluación contribuye al proceso de profesionalización de los enseñantes al permitirles abandonar el rol de técnicos que administran evaluaciones diseñadas por otros y adoptar el rol de personas que toman decisiones y que se guían por evaluaciones que ellos mismos han creado y dirigido.

En segundo lugar, ampliar el trabajo de enseñantes que practican evaluaciones fundamentadas requerirá más respeto y compromiso por parte de las personas ajenas al aula. Un enseñante, por muy consciente y dotado que sea, poca cosa podrá hacer solo por su cuenta. El apoyo a la evaluación basada en el enseñante debe darse tanto en la escuela misma como más allá de ella. Una evaluación efectiva requiere unas condiciones en las que los enseñantes puedan trabajar conjuntamente para establecer una visión sólida y compatida del currículo, para fijar objetivos claros para la enseñanza y fines para la evaluación, y para desarrollar un sistema coherente, que abarque toda la escuela, con el fin de evaluar el rendimiento de los estudiantes e informar sobre él.

Además, también debe darse un mayor reconocimiento, tanto en el seno de la comunidad educativa como de la sociedad en general, del rol crítico que pueden desempeñar los enseñantes en el proceso de evaluación. La mejor manera de alcanzar este reconocimiento es educando al público sobre las limitaciones de las pruebas estandarizadas y garantizando que el juicio cle los enseñantes sea una parte integral del programa de evaluación de cualquier estudiante.

En suma, la evaluación es fundamentada, en vez de informal, cuando se lleva a cabo por enseñantes conscientes que recurren a una variedad de estrategias para observar y documentar cuidadosamente el rendimiento de sus alumnos en diversos contextos y a lo largo del tiempo, mientras los estudiantes participan en auténticas tareas de aprendizaje. La evaluación fundamentada requiere profesionales conscientes y preparados en el aula, pero cada enseñante se encuentra limitado por lo que puede conseguir por su cuenta. 
Para que la evaluación basada en el enseñante alcance su potencial, también requiere un compromiso a nivel de escuela con formas alternativas de evaluación de los alumnos, junto con un mayor reconocimiento de la importancia del juicio del enseñante en el proceso de evaluación, tanto en el seno de la comunidad educativa como de la sociedad en general. Si se cumplen estas condiciones, la evaluación fundamentada realizada por los enseñantes puede ser un potente trampolín para mejorar la enseñanza y el aprendizaje en nuestras escuelas.

\section{Referencias}

ARChRALD, D. A. Y NEWMANN, F. M. (1988). Beyond standardized testing. Reston, VA: NASSP.

ATWEL, N. (1987). In the middle: Writing, reading, and learning with adolescents. Upper Monclair, NJ: Boynton/Cook.

BartLett, F. C. (1932). Remembering: A study in experimental and social psychology. Cambridge, Inghterra: Cambridge University Press.

BREWER, R. (1990). The development of portfolios in writing and, mathematics for state-wide axiessment in Vermont. Ponencia presentada en el Institute on New Modes of Assessment, Cambridge, $\mathrm{MA}$.

CALFEE, R. C. Y HiEBERT, E. H. (1989). Advancing academic literacy through teachers' assessments. Educational Leadership, 46, 50-54.

CANNEL, J. J. (1987). National norm-referenced elementary acbievement testing in America's public scbools: How all fifty states are above the national average. Charleston, WV: Friends of Education.

Goodman, Y. (1985). Kidwatching: Observing children in the classroom. En A. Jaggar y M. T. Smith-Burke (Eds.), Observing the language learner. (pp. 9-18), Urbana, Il y Newark, DE: National Council of Teachers of English and International Reading Association.

Goodman, Y. (1991). Informal methods of evaluation. En J. Flood, J. Jensen, D. Lapp y J. Squire (Eds.), Handbook of research on teacbing the English language arts. (pp. 502-509) Nueva York: Macmillan.

Graves, D. H. (1983). Writing: Teachers and children at work. Exeter, NH: Heinemann.

HAERTEL, E. (1990). Student achievement tests as tools of educational policy: Practices and consequences. En B. R. Gifford (Ed.), Test policy and test performance: Education, language, and culture. (pp. 13-14). Boston, MA: Kluwer.

НеAтH, S. B. (1983). Ways with words. Cambridge, Inglaterra: Cambridge University Press.

LIPSON, M. Y WIXSON, K. (1986). Reading disability research: An interactionist perspective. Review of Educational Research, 56, 111-136.

MCNeIL, L. (1988). Contradictions of control, Part 2: Teachers, students, and curriculum. Pbi Delta Kappan, 69, 433-438.

Miluer, G. A. (1956). The magical number seven, plus or minus two: Some limits on our capacity for processing information. Psychological Review, 63, 81-97.

PARIS, S. G., LAWTON, T. A, TURNER, J. C. Y ROTH, J. L. (1991). A developmental perspective on standardized achievement testing. Educational Researcher, 20, 12-20.

Pearson, P. D. (1984). Direct explicit teaching of reading comprehension. En G. Duffy, L. Roehler y J. Mason (Eds.), Comprebension instruction: Perspectives and suggestions (pp. 222-233). Nueva York: Longman.

Report of the National Commission on Testing and Public Policy. (1990). From gatekeeper to gateway: Transforming testing in America. Chestnut Hill, MA: Boston College.

SMrTH, M. L. (1991). Put to the test: The effects of external testing on teachers. Educational Researcher, $20,8-11$.

Sticilins, R. J. (1985). Improving assessment where it means the most: In the classroom. Educational Leadership, 43, 69-74.

Sticigins, R. J. (1991). Assessment literacy. Phi Delta Kappan, 72, 534-539.

TAYLoR, D. (1989). Toward a unified theory of literacy learning and instructional practices. Phi Lelta Kappan, 71, 184-193.

VALENC:A, S. y CAIFEE, R. (1991). The development and use of literacy portfolios for students, classes, and teachers. Applied Measurement in Education, 4, 333-345.

Valencia, S., McGinley, W. y Pearson, P. D. (1990). Assessing literacy in the middle school. En G. Duffy (Ed.), Reading in the middle scbool ( $2^{a}$ Ed.), Newark, DE: International Reading Assiciation.

Winecirad, P. Y ParIs, S. G. (1989). A cognitive and motivational agenda for reading instruction. Educational Leadership, 46(4), 30-36.

WoLf, D. P. (1989). Portfolio assessment: Sampling student work. Educational Leadership, 46, $35-39$ 


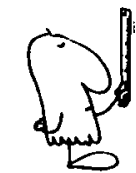

De la evaluación informal

a la evaluación fundamentada:

Reconocer el rol del enseñante en clase

Kenneth P. Wolf

CL\&E, 1993, 19-20, 35-42

Datos sobre el autor: Kenneth P. Wolf enseña en la University of Northern Colorado.

Articulo original: From informal to informed assessment: recognizing the role of the classroom teacher. En Journal of Reading, Vol. 36, № 7, pp. 518 523. Traducción de Genis Sánchez. Reproducido con autorización de Kenneth P. Wolf y de la International Reading Association (La I.R.A. no se responsabiliza de la adecuación de la traducción)

Dirección : 5579 Mesa Top Court, Boulder, CO 80301, EEUU.

(C) De todos los artículos deberá solicitarse por escrito autorización de CL\&E y de los autores para el uso en forma de facsímil, fotocopia o cualquier otro medio de reproducción impresa. CL\&E se reserva el derecho de interponer acciones legales necesarias en aquellos casos en que se contravenga la ley de derechos de autor. 